\title{
Assuring the information flow from accident sites to decision makers - a Finnish case study
}

\author{
R. Molarius ${ }^{1}$, J. Korpi ${ }^{2}$, H. Rantanen ${ }^{3}$, H. Huovila ${ }^{1}$, J. Yliaho ${ }^{1}$, \\ N. Wessberg ${ }^{1}, \mathrm{~K}$. Virrantaus ${ }^{2} \& \mathrm{~V}$. Rouhiainen ${ }^{1}$ \\ ${ }^{1}$ VTT Technical Research Centre of Finland, Finland \\ ${ }^{2}$ TKK Technical University of Helsinki, Finland \\ ${ }^{3}$ Emergency Services College, Finland
}

\begin{abstract}
In spite of the huge amount of information regarding natural and man-made accidents, rescue forces are not able to use information efficiently in their decision-making. From a rescue services point of view, the main challenge is not only to get authentic and real data, but to get ready analysed data. This paper discusses the Finnish way to create an emergency management system that collects reasonable data from an emergency situation, merges the data to get a better overall picture, and sends the analysed assessment of the situation to the rescue services and decision-makers. Two pilot tests were made to define how the information would flow from accident sites to rescue authorities and decision-makers in the best way. The results show that a network has to be established for supplying and analysing the information into a suitable form to be used in real-time management of crisis situations. The authorities also have to ensure that there are no technical limits which obstruct the real-time information flow from accident sites to the decision-makers' monitors.
\end{abstract}

Keywords: common operational picture, visualization, incident.

\section{Background}

All rescue organisations today advance the management of severe accidents and disasters more effectively, and therefore the common operational picture (COP) has emerged as a reformer of methods. The term 'common operational picture' is associated with gaining "an overview of an incident by all relevant parties that provides incident information enabling the Incident Commander and any 
supporting agencies and organisations to make effective, consistent, and timely decisions" [1]. The COP rarely mirrors the common operating understanding of the situation but these systems can collect the data from accident sites and transform them into rescue services.

From a rescue services point of view, the main challenge today is not only to get authentic and real data, but to get ready analysed data. The lack of widely accepted standards and performance issues creates a large number of different formats and raises barriers in data fusion and communication between authorities. Besides a huge amount of data, the problem in large-scale incidents is communication, due to different technical systems, operations models, and cultural differences.

Nowadays, analysis and visualization tools exist for virtually every kind of Earth-Observation data. However, what often occurs is that these tools are "self contained", and mixing data that comes from different sources can be unsure, unverified, and useless at least for its purpose. Furthermore, this occurs much more frequently when real-time visualization is involved. For rescue services' use, the received data must be perfect. Hence, we focused on analysing the data in the most suitable form for different end-users. This will not let us support realtime visualisation from all the available data, but it will give an analysed and trustworthy visualisation for common operational pictures. This will also minimise the risk of false data.

This paper focuses on the framework called MANAGE, (MANAGing the Emergencies), which improves the stages of an emergency response system from Outbreak to Informing the public and media. The framework is designed for and piloted in the Finnish rescue environment but the basic idea is meant to be applicable in the other countries, too. It will make the information flow more fluently between rescue authorities, contributory authorities, experts, media, and common citizens pending the accidents; and moreover, it will produce readyanalysed information for decision-makers.

In the next sections, we will first present the framework of MANAGE. After that, we will clarify two procedures on how to analyse raw material for rescue service use. The first elements of the MANAGE is made for catching weak signals, i.e., to prioritise the most dangerous chemical sites according to the vulnerability of their environment. The second element is a spatio-temporal population model that produces the whereabouts of citizens in emergencies. The visualization methods used in the MANAGE are introduces in the next chapter and finally, we will discuss future development targets in Finland.

\section{The incident procedure in Finland}

Every single accident includes the same stages: weak signals, early warning, outbreak, observation, emergency response, and response activities. For example, chemical accidents are distinguished from acute or current risks by the fact that there are already some indications of them that have not yet materialized into a clearly definable or even quantifiable threat. It is plausible that there exist weak signals that might be discovered [2]. Early Warning is usually the first visible sign that will tell that something undesirable has taken place. If this 
knowledge leads to favourable action, it is possible to diminish the consequences of the accident or in the best case even prevent the accident.

The period of time when the accident starts may be appointed as Outbreak, which is usually a very short moment before the Observation takes place. From the point of view of rescue operations, it is vital to lengthen the time between Early Warning and Outbreak. On the other hand, the time period between the Outbreak and Observation should also be shortened.

When the chemical accident is observed, the observer informs the Emergency Response Centre, which will make the initial analysis and dispatch. The rescue services carry out Response Activities, such as the selection of proper prevention activities. Therefore they need information about the released chemical, drift of the chemical plume, and people in danger. It is also essential that shared information will at best change the sequential order of actions to parallel actions that will enhance the use of resources and save time in critical moments. This is where the COP systems are necessities.

Today's IT tools are of great help in many cases. However, a great many of the issues dealing with human computer interfaces are problematic in the state of incidents where there is the need to observe, understand, and integrate a wide range of information in a very limited time and in adverse conditions. Human perception is often insufficient, and therefore plenty of effort should be made to change the information accessible and to avoid information overload [3].

\section{Framework of MANAGE}

The MANAGE distributed visualising platform with open architecture was developed to ensure collaborative data information and visualization for authorities and other involved parties connected using their own familiar equipment and tools. It also highlights the role of experts while the emergency is on. Because of the fast actions at emergencies, rescue authorities have no possibility to analyse the incoming data. The data must be readily analysed into the form according to recipients' needs. For example, rescue services do not need the data dealing with the concentration of the chemical plume, but they need information about The Emergency Response Planning Guideline values (ERPG), which provide estimates where one reasonably might anticipate observing adverse effects.

The framework of MANAGE is seen in Figure 1. The heart of MANAGE is a server that collects, arranges, and transmits information between the parties.

The heart of MANAGE also includes all the required and necessary models and methods to analyse the raw data and illustrate the analysis results in a suitable form. In our pilot case, this contains the vulnerability analysis, dispersion model, and spatio-temporal population model. MANAGE merges all the received raw data into a developed analysis and models, and the relevant analysed data is then conveyed to the appropriate emergency response actors and stakeholders.

The MANAGE framework also includes methods to collect and produce raw data like sensor network systems with wireless sensor network modules, 


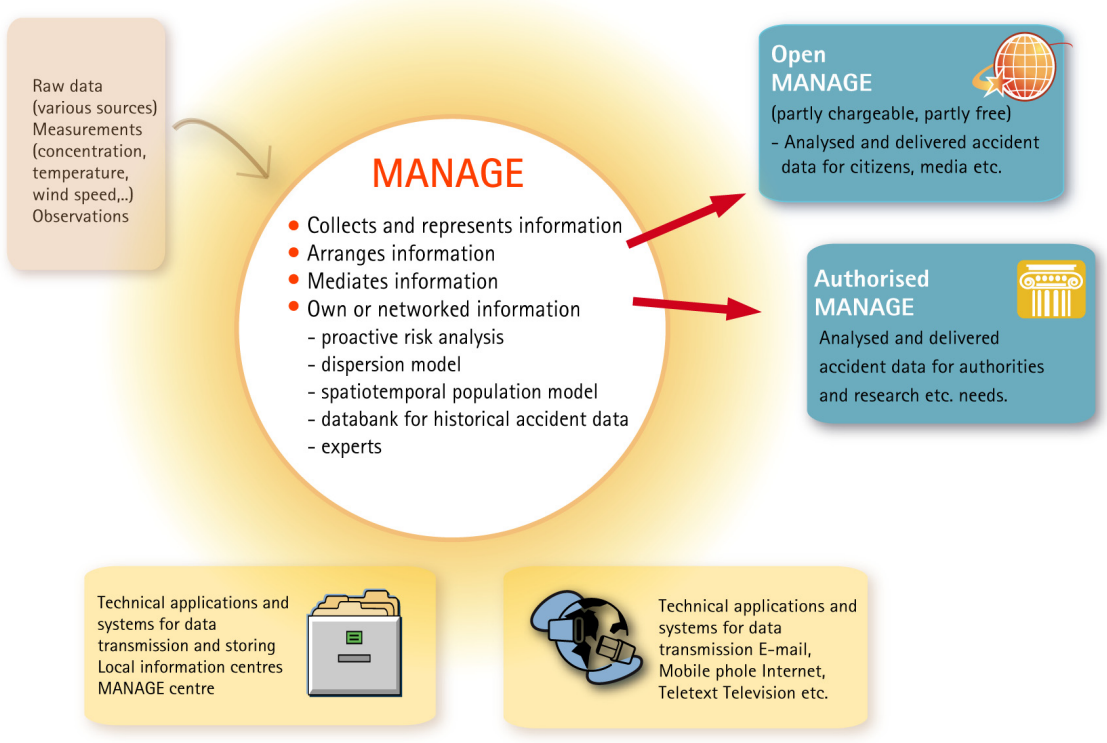

Figure 1: $\quad$ The framework of MANAGE.

including chemical sensors and temperature sensors at the chemical site. In our pilot case, the measured data is gathered through the coordinator node to a laptop computer, which transfers the data to a remote database via a $3 \mathrm{G}$ data connection.

The MANAGE includes two portals for end-users. The Open Manage provides both chargeable and free information from accident sites to media and citizens. The Authorised Manage provides information for rescue authorities, police, hospitals, etc. A WMS interface for requesting metadata and multi-layer map images was incorporated into the pilot system.

The Open Manage provides business opportunities for the owners of the COP system. Besides equipment vendors and knowledge/telephone operators, business can be created by selling analysed additional knowledge about the accident for instance to media or citizens. The historical accident data bank would be valuable to researchers, companies or other stakeholders, who may be willing to pay for such knowledge.

\section{Promoting analyses and models}

MANAGE promotes analysis and models that process the raw data for the use of end-users. The elements we developed were the vulnerability analysis to work out the weak signals and spatio-temporal population model together with the dispersion model. 


\subsection{Vulnerability analysis}

The framework for the vulnerability analysis was developed in cooperation with the Finnish chemical authorities and the Finnish rescue services. The goal of the study was to develop a prognostic method vulnerability analysis of the chemical industry for the chemical authorities. The method helps authorities to prioritise the most significant chemical targets where chemical spills may result in the most severe consequences to the citizens or environment.

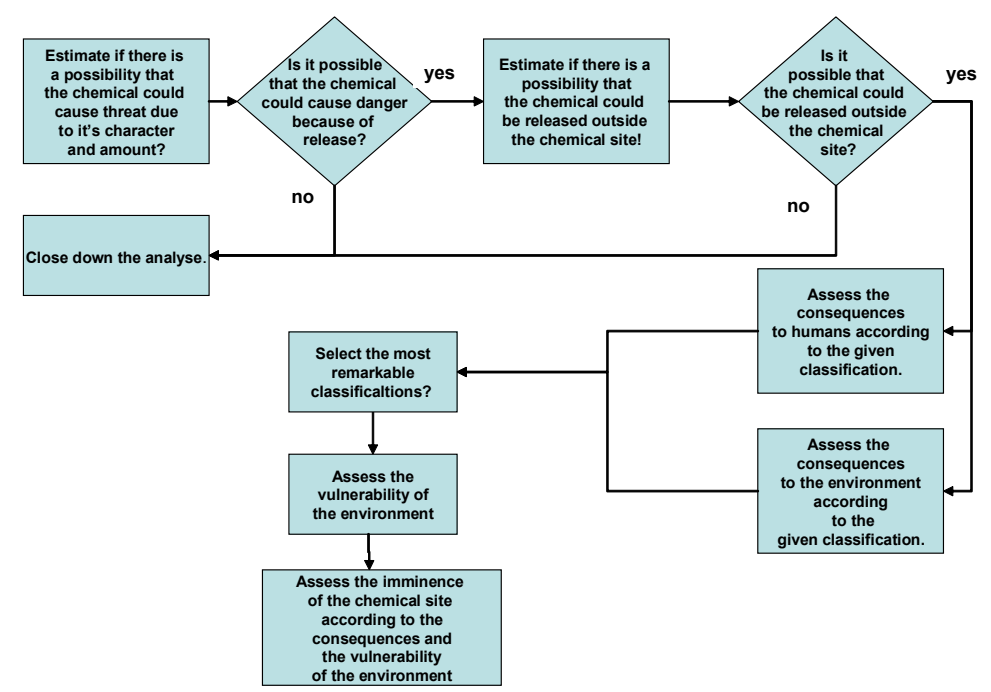

Figure 2: The framework for vulnerability analysis.

The vulnerability analysis is based on an environmental risk analysis developed by VTT in 2005 [4]. The framework of vulnerability analysis for society and the environment is shown in Figure 2. It was evolved through various brainstorming sessions with rescue services and chemical authorities. The method is documented in Finnish in VTT Report 2009 [5].

\subsection{Spatio-temporal population model and dispersion model}

The leading idea when producing the spatio-temporal model was that the model should be flexible enough to be suitable for every municipality in Finland [6].

To create the spatio-temporal population model, two main data sources were used in modelling the population, the Finnish Population Information System and Digiroad. The Finnish Population Information System is a national register that contains basic information about citizens and is maintained by the national population register centre. Also recorded is information about buildings, construction projects, residences, and real estate. The building information includes, e.g., location coordinates, gross floor area, number of stores, purpose of use, and number of residents. 
Digiroad is a national road and street database administered by the Finnish Road Administration. The database contains information on the geometry and physical features of roads and streets in Finland. One attribute in the data is the traffic volume, which reports the average number of vehicles passing a road segment per day.

The risk area for the population model is defined with the ESCAPE dispersion model (Expert System for Consequence Analysis using a PErsonal computer) developed by the Finnish Meteorological Institute [7]. The ESCAPE models the atmospheric dispersion of hazardous substances in a chemical release. The ESCAPE result gives the Emergency Response Planning Guideline (ERPG) values to define the risk area from where the population model needs to be calculated. The ERPG values provide estimates of concentration ranges of detrimental effects as a consequence of exposure to the specific substance.

Due to the available data, an object-oriented spatio-temporal model is used for implementing the model. In an object-oriented spatio-temporal model, the real world is modelled with the help of individual spatio-temporal atoms, which have a temporal dimension [8]. Real-world objects, such as buildings and roads, are divided into different categories, and each category is one spatio-temporal atom. For our case, 11 spatio-temporal atoms are defined, e.g., roads, office buildings, old people's homes, day-care centres, shops, hospitals, and schools. For each spatio-temporal atom, the population size changes individually according to time. The population in an object at a certain time is defined according to a time use table and is expressed as a percentage of the maximum or the average. Time use table is based on time use surveys. The developed spatiotemporal model is also a knowledge-based model, since parameters are defined on the basis of the user's knowledge. For each object (road segment or building), the maximum population is defined.

The population model produces two output formats. The size of the population inside the affected area is given as a numeric text report. If the data provides the possibility, the model can differentiate the people needing special attention, for example, elderly people and children, and give the share of special attention people from the total population in the affected area. Also, the size of the population inside affected areas of different degrees can be specified in the report.

The ESRI shape file is an output format that enables a more thorough analysis of the population distribution inside the affected area. The coordinates of the individual objects and the estimated populations in those objects are stored in the shape file. The spatial distribution of population inside the affected area is especially useful in the allocation of rescue resources if the affected area is large.

\section{Visualization the MANAGE results}

In MANAGE, both static and dynamic information is visualized. Also, outputs of different models are visualized. Although much of the visualized information is dynamic, only static methods are used, and animations are excluded because the animations easily become too complex to be accurately perceived [9]. In 
situation picture systems like MANAGE, where several model outputs are visualized at once, the information is too dense for animations.

Static information visualized in a MANAGE situation picture includes the infrastructure, geographical environment, and the result of the vulnerability analysis. The vulnerability analysis produces information about the possible consequences of a chemical release, taking into account both the characteristics of the environment and the characteristics of the chemical. The vulnerability analysis is done as part of preparedness in the time of steady-state conditions, so it is static information.

Dynamic information visualized in a MANAGE situation picture includes the incident location information and two model outputs, a dispersion model and a population model. The dispersion model models the atmospheric dispersion of the released chemical substance. The population model models the population distribution over the risk area at the time when the chemical release takes place.

The background map functions as a spatial reference for the situation information and includes information about the physical environment and infrastructure inside the area of interest. The background map in a situation picture map should be of a faded design in order to allow enough prominence to the dynamic situation information [10]. The map should also be scalable in order to be readable at various scales, and thus vector map formats are preferred. The background map of a situation picture map needs to also include additional information compared to a basic topographic map or city map. Establishments that may have special people in them, such as elderly people and children, should be visible in the background map. Also, locations of the resources of emergency authorities could be highlighted in the background map.

The result of the vulnerability analysis shows the radius of the risk area according to national guidelines for rescue actions in case of an emergency. The result of the vulnerability analysis also shows the degree of criticality of the possible release to the environment, on a scale of seven, with 1 being the most severe. The visualization is a circle shape polygon around the possible source of release. The colour of the polygon depends on the vulnerability class. It is highly transparent in order to allow visibility for the background map and for the dynamic situation information. The location of the possible source of release is visualized by using the warning category of SHIFT symbols. SHIFT symbols are a set of culturally independent map symbols that are suitable in information exchange in a wide range of different crises, disasters, and emergency situations [11].

The result of a dispersion model of a chemical release is visualized by nested polygons in MANAGE, where the ERPG values isolines are shown, see Figure 3. This makes it possible to put different chemicals on the same scale and visualize a risk area according to its consequences for humans. This again helps to directly see the magnitude of the situation at hand.

There are three different ERPG values defined. Three degrees of affected areas are enough to visualize the variation in the magnitude but still be easy to perceive from a cluttered situation picture map. Polygons are visualized with colours that do not include the colours used in a visualization vulnerability 


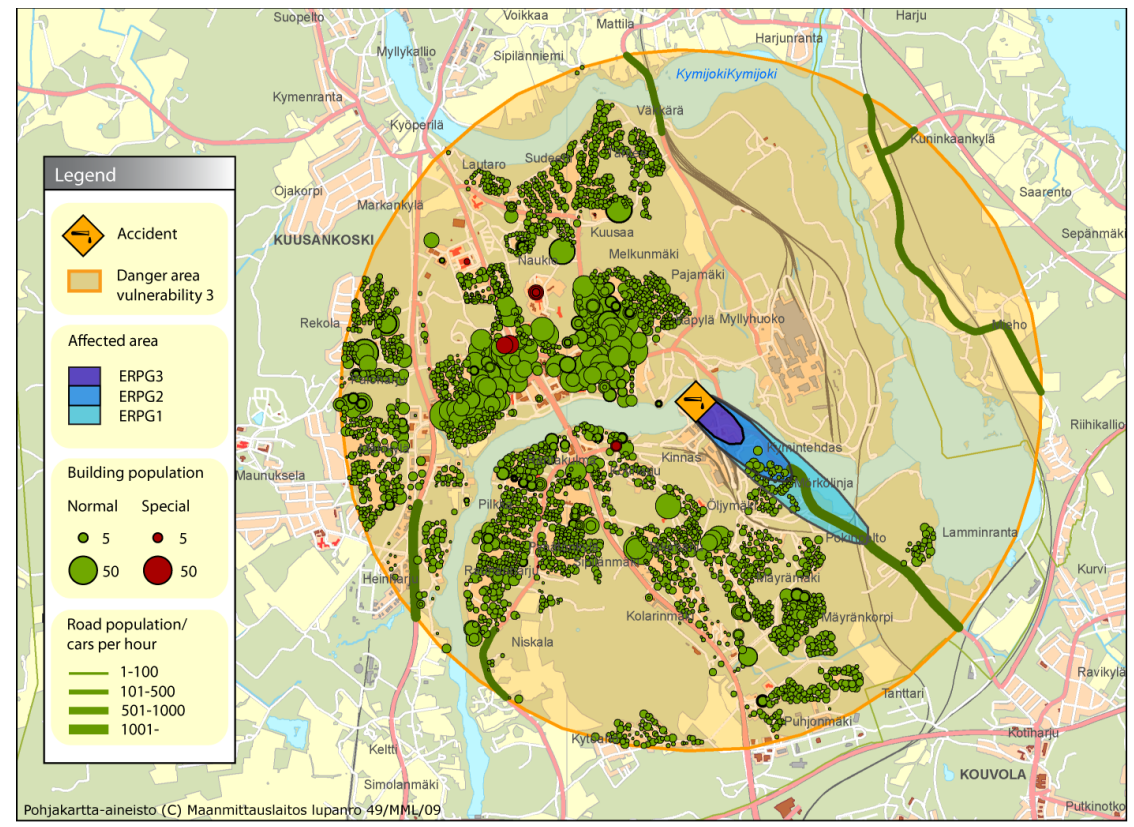

Figure 3: $\quad$ MANAGE situation picture map.

analysis. In small scale, the polygons are solid but become more transparent when a smaller area is viewed.

The result of the population model is visualized by the proportional symbols method. Each population point, i.e., a building or a road segment, is visualized by one symbol. The size of the symbol is proportional to the population estimated in that location. Humans are very good at recognizing the variation in phenomena visualized with concentrations of symbols. The use of a colour scale would also visualize the variation, but adding another colour fill would make the situation picture map unreadable, whereas proportional symbols also allow the background map to be shown.

For roads, the most natural choice for a symbol is a basic line symbol with a varying line thickness indicating the variation in population. For buildings, the basic circle symbol proved to be best. Buildings with potentially embodying special attention people can be identified with the use of different symbol colour. Only the overview of the population distribution can be visualized by proportional symbols. Exact information about the total number of people inside a risk area must also be given.

\section{Discussion and conclusions}

The results show that it is possible to build a system that will produce the ready analysed information to rescue services and provide it for their use already when the chemical accident is on. While the alarm reaches rescue services via the 
Emergency Response Centre, the chemical data from the accident site has left to the dispersion modellers and spatiotemporal modellers. Moreover, at the same time when the rescue services enter the accident site, the modellers count the ERPG of the plume and analyse where the citizens are. If the net works well, the information will be in use just in time when the rescue services are at the emergency site. The spatial distribution of population inside the affected area is especially useful in allocation of rescue resources when the affected area is large.

However, there are some constraints on the system dealing with the organisation of expert institutions. Firstly, there must be contracts between society and expert institutions about the 24/7 working hours and emergency readiness. If not, there is no use for the swift information flow from the accident site to the model experts because most of the time goes by while looking for the experts.

Secondly, the quick, online models must be in use and the background information for models has to be available, too. It would be desirable if the authorities will produce the vulnerability analysis from all chemical targets and hence this, oblige the most important sites to connect this kind of information system with their own sensor networks. This would enable the expert institutions to collect the required background information for safety.

It must be noticed that today, before the model is in use, one professional worker is needed to set up the environment and to parameterize the model to local conditions. The worker should also be familiar with the Java programming language and basic GIS tools and have knowledge of the characteristics of the city.

\section{References}

[1] Glossary of FEMA (Federal Emergency Management Agency) of the US government tasked with Disaster Mitigation, Preparedness, Response and Recovery planning. http://www.w3.org/2005/Incubator/eiif/wiki/Fema

[2] Rantanen, H., Coping with Power Disturbances. Proc of Nordregio 2008. Early Warning and Civil Protection - When does it work and why does it fail? Eds. C. Pursiainen \& P. Francke. Nordregio Report 1:2008. Stockholm, Sweden, pp 96-119, 2008

[3] Rantanen H., Managing Emergency Response with the Help of Information Technology. A Feasibility Study in the Finnish Perspective, Licentiate thesis, University of Kuopio, Finland, 2003.

[4] Wessberg, N., Molarius, R., Seppälä, J., Koskela, S. \& Pennanen, J., Environmental risk analysis for accidental emissions. Journal of Chemical Health \& Safety, vol.15, no.1, January/February pp.24-32, 2008. doi-link: http://dx.doi.org/10.1016/j.jchas.2007.07.001.

[5] Molarius, R., Nissilä, M., \& Virolainen K., The method for assessing the threat for citizens and environment due to chemical sites. In Finnish. VTT Report VTT-R-02167-09. Tampere, Finland, 2009. 
[6] Zhang, Z., A spatio-temporal Population model for alarming, situation picture and warning system. Masters degree thesis, Helsinki University of Technology. Helsinki, Finland, 2009

[7] Riikonen, K., Nikmo, J. \& Kukkonen, J., A validated assessment tool for consequence analysis of chemical emergencies - ESCAPE for Windows. Proc. of the NBC 2000 Symposium on nuclear, biological and chemical threats in the 21st century, Eds. E. Kujala, K. Laihia \& K. Nieminen, Espoo, Finland. 286-287, 2000.

[8] Nadi, S., \& Delavar, M.R., Spatio-temporal modeling of dynamic phenomena in GIS. Proc of the 9th Scandinavian Research Conference on Geographical Information Science Eds. Virrantaus K \& Tveite H, 2003, Espoo, Finland. pp. 215 - 225, 2003.

[9] Tversky, B., Morrison, J.B. \& Betancourt, M. Animation: can it facilitate? International Journal of Human-Computer Studies, 57 , pp. 247-262. 2002.

[10] Salonen, T., Development of visualization for the military situation picture and the combat model. Masters degree thesis, Helsinki University of technology. Finland, 2005.

[11] Korpi, J., Designing pictorial symbols for situation pictures of international crisis management. Towards Better Situational Awareness, eds. J. Jormakka \& S. Oksa, Finnish Defense University, Helsinki, Finland, pp 1126,2008 . 